\title{
Existence of Adaptively Stable Sunspot Equilibria near an Indeterminate Steady State*
}

\author{
George W. Evans \\ University of Oregon
}

\author{
Seppo Honkapohja \\ University of Helsinki
}

April 6, 2002

\begin{abstract}
We examine the nonlinear model $x_{t}=E_{t} F\left(x_{t+1}\right)$. Markov SSEs exist near an indeterminate steady state, $\hat{x}=F(\hat{x})$, provided $\left|F^{\prime}(\hat{x})\right|>$ 1. Despite the importance of indeterminacy in macroeconomics, earlier results have not provided conditions for the existence of adaptively stable SSEs near an indeterminate steady state. We show that there exist Markov SSEs near $\hat{x}$ that are E-stable, and therefore locally stable under adaptive learning, if $F^{\prime}(\hat{x})<-1$.
\end{abstract}

JEL classifications: C62, D83, D84, E31, E32

Key words: Indeterminacy, learnability, expectational stability, endogenous fluctuations

\section{Introduction}

Existence of stationary sunspot equilibria (SSEs) has received widespread attention since the initial investigations of (Shell 1977), (Azariadis 1981) and (Cass and Shell 1983). ${ }^{1}$ An important case of SSEs are those that occur

*Financial support from the US National Science Foundation, Academy of Finland, Yrjö Jahnsson Foundation and Nokia Group is gratefully acknowledged.

${ }^{1}$ Recent surveys are provided by (Chiappori and Guesnerie 1991) and (Guesnerie and Woodford 1992). 
near an "indeterminate" steady state, i.e. a steady state such that there exists a continuum of perfect foresight paths converging to it. (Chiappori, Geoffard, and Guesnerie 1992) show for a general class of models that a continuum of finite state Markov SSEs invariably exists in any neighborhood of such a steady state. The case of indeterminate steady states is also of particular interest since it is the starting point for the current generation of macroeconomic models based on self-fulfilling prophecies. This recent body of literature is surveyed in (Benhabib and Farmer 1999) and (Farmer 1999).

In the context of the standard Overlapping Generations model (Woodford 1990) raised the issue of whether agents would be able to coordinate on SSEs, if they followed adaptive learning rules, and showed convergence to some SSE when the steady state is indeterminate. Woodford's argument was based on global analysis using the index number theorem of Poincare-Hopf and the structure of the invariant set under learning dynamics. This approach does not provide any information about the location of the stable sunspots and in particular about whether the SSEs near the steady state are stable or unstable under learning.

Analysis of local stability of finite state SSEs under adaptive learning for a general class of models was undertaken in (Evans and Honkapohja 1994). ${ }^{2}$ Convergence conditions under adaptive learning dynamics were shown to be governed by Expectational Stability (or "E-stability") conditions. It was also shown that sharp stability results are available for SSEs near deterministic cycles or near a set of distinct steady states. ${ }^{3}$ However, in the case of SSEs near a single steady state, the stability results have heretofore remained incomplete.

In this paper we complete the analysis of adaptive stability of SSEs near a single steady state in the general univariate model $x_{t}=E_{t} F\left(x_{t+1}\right){ }^{4}$ For this model indeterminacy of a steady state $\hat{x}$ corresponds to $\left|F^{\prime}(\hat{x})\right|>1$. In (Evans and Honkapohja 1994) we showed that if $F^{\prime}(\hat{x})>1$ then every finite state SSE in a small enough neighborhood of $\hat{x}$ is unstable under learning. However, stability for the case $F^{\prime}(\hat{x})<-1$ remained an open issue. Using

\footnotetext{
${ }^{2}$ Part IV of (Evans and Honkapohja 2001) provides an overview of learning of rational expectations equilibria in nonlinear models.

${ }^{3}$ (Howitt and McAfee 1992) and (Evans, Honkapohja, and Romer 1998) exploit this type of result for the case of multiple steady states.

${ }^{4}$ Eductive approaches can also be used to investigate coordination on rational expectations equilibria. Eductive stability of SSEs in an overlapping generations model is examined in (Desgranges and Negroni 2001).
} 
local bifurcation theory we are here able obtain sharp stability results for this remaining case: there always exist nearby Markov SSEs that are stable under adaptive learning if $F^{\prime}(\hat{x})<-1$.

\section{The Framework}

We look at the univariate model

$$
x_{t}=E_{t}^{*} F\left(x_{t+1}\right),
$$

where $E_{t}^{*} F\left(x_{t+1}\right)$ denotes the expectation of $F\left(x_{t+1}\right)$ held by agents at time $t$. Under the rational expectations assumption $E_{t}^{*} F\left(x_{t+1}\right)=E_{t} F\left(x_{t+1}\right)$, the true mathematical conditional expectation of $F\left(x_{t+1}\right)$ based on information at time $t$. We assume throughout that there exists a steady state $\hat{x}=F(\hat{x})$, that $F$ is three times continuously differentiable in a neighborhood of $\hat{x}$, that $F^{\prime}(\hat{x}) \neq 0$, and that $F^{\prime \prime}(\hat{x}) \neq 0 .^{5}$

For the purposes of this paper it will be sufficient to examine 2-state SSEs. We therefore focus on this case, commenting where appropriate on how our results extend to the more general finite state case. A two-state Markov SSE is a stochastic process for $x_{t}$, depending on an exogenous two-state Markov process $s_{t}$ with transition probability matrix $\Pi=\left(\pi_{i j}\right)$, which satisfies the above equation (1) with rational expectations. Let the two states of $s_{t}$ take the values $s_{t} \in\{1,2\}$. Then an SSE takes the form

$$
x_{t}=\bar{x}_{i} \text { if } s_{t}=i, \text { for } i=1,2,
$$

with $\bar{x}_{1} \neq \bar{x}_{2}$ and satisfying

$$
\begin{aligned}
& \bar{x}_{1}=\pi_{11} F\left(\bar{x}_{1}\right)+\left(1-\pi_{11}\right) F\left(\bar{x}_{2}\right), \\
& \bar{x}_{2}=\left(1-\pi_{22}\right) F\left(\bar{x}_{1}\right)+\pi_{22} F\left(\bar{x}_{2}\right) .
\end{aligned}
$$

It is well known that such solutions exist in a neighborhood of the steady state $\hat{x}$ when $\left|F^{\prime}(\hat{x})\right|>1$. Existence and characterization of SSEs has been examined using a variety of approaches. For a review, see (Chiappori and Guesnerie 1991). As noted above, the stability results for the SSEs near an indeterminate steady state have been incomplete, even though indeterminate steady states and nearby SSEs play a major role in the literature.

\footnotetext{
${ }^{5}$ The linear case is analyzed at length in a companion paper (Evans and Honkapohja 2002).
} 


\subsection{Adaptive Learning}

To analyze the system under adaptive learning, we consider agents who believe they are in a 2-state SSE with unknown values to be taken by $x_{t+1}$ in the two sunspot states. A standard estimator for these values is given by state contingent averaging, i.e. they are estimated as the average past value taken by $x_{t}$ in each state.

Formally, let $\phi_{t}=\left(\phi_{1 t}, \phi_{2 t}\right)$ be the estimates of the values that $x_{t}$ takes in states 1 and 2 of the sunspot. Let also $\psi_{j t}=1$ if $s_{t}=j$ and 0 otherwise be the indicator function for state $j$ of the sunspot. Clearly, $\psi_{2 t}=1-\psi_{1 t}$. Then we can write the learning rules based on state-contingent averaging in the following form:

$$
\begin{aligned}
\phi_{j t} & =\phi_{j, t-1}+t^{-1} \psi_{j, t-1} q_{j, t-1}^{-1}\left(x_{t-1}-\phi_{j, t-1}+\varepsilon_{t-1}\right) \\
q_{j t} & =q_{j, t-1}+t^{-1}\left(\psi_{j, t-1}-q_{j, t-1}\right) \\
x_{t} & =\psi_{1 t}\left[\pi_{11} F\left(\phi_{1 t}\right)+\left(1-\pi_{11}\right) F\left(\phi_{2 t}\right)\right]+\psi_{2 t}\left[\left(1-\pi_{22}\right) F\left(\phi_{1 t}\right)+\pi_{22} F\left(\phi_{2 t}\right)\right]
\end{aligned}
$$

for $j=1,2$. We note here that in the learning rules agents are assumed to use observations only through period $t-1$.

These equations are interpreted as follows. $t q_{j, t-1}$ is the number of times state $j$ has occurred up to time $t-1$. The second equation is the recursion for the fraction of observations of state $j$. The first equation is then a recursive form for the state averages, including a small measurement or observation error $\varepsilon_{t-1} \cdot{ }^{6}$ Finally, the third equation gives the temporary equilibrium for the model, since the right-hand side is the expectation of the value of $F\left(x_{t+1}\right)$ given the forecasts $\phi_{j t}$. Note also that, for the sake of simplicity, the agents are assumed to know the transition probabilities $\pi_{i j}$.

As established in the earlier literature, stability under adaptive learning is governed by E-stability. For the current framework the connection is established in Section 5 of (Evans and Honkapohja 1994), see also (Evans and Honkapohja 2001), Chapter 12. In the remainder of this paper we therefore derive E-stability results since these are necessary and sufficient for local stability under adaptive learning.

\footnotetext{
${ }^{6} \varepsilon_{t}$ is assumed to be iid mean zero and to have a bounded support. The measurement error is needed only for the instability result, see (Evans and Honkapohja 1994) for further details.
} 


\section{$2.2 \quad$ E-Stability}

E-stability is defined in terms of the mapping from a corresponding perceived law of motion (PLM) to the implied actual law of motion (ALM). At this stage it is convenient to switch back to the notation in which $x_{i}$ denotes the perceived value of the endogenous variable in sunspot state $i$. The PLM then takes the form $x_{t+1}=x_{i}$ if $s_{t+1}=i$, for $i=1,2$. Under the PLM, expectations are calculated to be $E_{t}^{*} F\left(x_{t+1}\right)=\pi_{i 1} F\left(x_{1}\right)+\pi_{i 2} F\left(x_{2}\right)$ if $s_{t}=i$. From (1) we then obtain the implied actual law of motion $x_{t}=\pi_{i 1} F\left(x_{1}\right)+\pi_{i 2} F\left(x_{2}\right)$, for $s_{t}=i$, so that the mapping from PLM to ALM parameters is given by

$$
T\left(\begin{array}{l}
x_{1} \\
x_{2}
\end{array}\right)=\Pi\left(\begin{array}{c}
F\left(x_{1}\right) \\
F\left(x_{2}\right)
\end{array}\right)
$$

The differential equation defining E-stability is

$$
\frac{d}{d \tau}\left(\begin{array}{l}
x_{1} \\
x_{2}
\end{array}\right)=T\left(\begin{array}{l}
x_{1} \\
x_{2}
\end{array}\right)-\left(\begin{array}{l}
x_{1} \\
x_{2}
\end{array}\right),
$$

where $\tau$ denotes notional or virtual time. If the equilibrium point $\left(\bar{x}_{1}, \bar{x}_{2}\right)^{\prime}$ is locally stable under this differential equation then it is said to be E-stable. Note that the equilibria of this differential equation consist of the steady state $(\hat{x}, \hat{x})^{\prime}$ as well as the possible SSEs.

Writing the differential equation explicitly

$$
\frac{d}{d \tau}\left(\begin{array}{l}
x_{1} \\
x_{2}
\end{array}\right)=\left(\begin{array}{cc}
\pi_{11} & 1-\pi_{11} \\
1-\pi_{22} & \pi_{22}
\end{array}\right)\left(\begin{array}{c}
F\left(x_{1}\right) \\
F\left(x_{2}\right)
\end{array}\right)-\left(\begin{array}{c}
x_{1} \\
x_{2}
\end{array}\right),
$$

E-stability conditions are obtained by linearizing this system at the equilibrium. Thus, an SSE $\left(\bar{x}_{1}, \bar{x}_{2}\right)$ is E-stable if the eigenvalues of the matrix

$$
\left(\begin{array}{cc}
\pi_{11} & 1-\pi_{11} \\
1-\pi_{22} & \pi_{22}
\end{array}\right)\left(\begin{array}{c}
F^{\prime}\left(\bar{x}_{1}\right) \\
F^{\prime}\left(\bar{x}_{2}\right)
\end{array}\right)-I
$$

have negative real parts. Here $I$ is the $2 \times 2$ identity matrix.

For SSEs nearby a steady state some useful results are implied by analysis of the linearization at the steady state, i.e.

$$
\left(\begin{array}{c}
\dot{w}_{1} \\
\dot{w}_{2}
\end{array}\right)=\left(F^{\prime}(\hat{x}) \Pi-I\right)\left(\begin{array}{c}
w_{1} \\
w_{2}
\end{array}\right)+\left(\begin{array}{l}
\Psi_{1}\left(w_{1}, w_{2}\right) \\
\Psi_{2}\left(w_{1}, w_{2}\right)
\end{array}\right),
$$


where $w_{i}=x_{i}-\hat{x}$ and $\dot{w}_{i}=d w_{i} / d \tau$, and where $\Psi_{i}\left(w_{1}, w_{2}\right)$ denote the nonlinear parts, $i=1,2$. Since $\Pi$ is a probability matrix, its eigenvalues are 1 and $\pi_{11}+\pi_{22}-1$. Thus the eigenvalues of the linear map are $F^{\prime}(\hat{x})-1$ and $F^{\prime}(\hat{x})\left(\pi_{11}+\pi_{22}-1\right)-1$. We immediately have the following result by continuity of eigenvalues:

Proposition 1 If $F^{\prime}(\hat{x})>1$, then every SSE sufficiently near the steady state fails to be E-stable.

This result was obtained earlier in (Evans and Honkapohja 1994). We remark that the proposition in fact holds for all $K$-state Markov SSEs, with $K \geq 2$, since every $K \times K$ probability matrix has an eigenvalue of 1 .

On the other hand, if $F^{\prime}(\hat{x})<-1$ then the existence or non-existence of E-stable SSEs is not obvious. While the E-stability condition based on (4) can still be used to check whether a given SSE near $\hat{x}$ is E-stable, there is no simple way to determine whether stable cases exist. Based on the linearization at the steady state, the eigenvalues will be near $F^{\prime}(\hat{x})-1$ and $F^{\prime}(\hat{x})\left(\pi_{11}+\pi_{22}-1\right)-1$. Clearly, the root near $F^{\prime}(\hat{x})-1$ is stable, but the possible values for the other root are not self-evident. In fact, as we show below, there are SSEs near the steady state with values for $F^{\prime}(\hat{x})\left(\pi_{11}+\right.$ $\left.\pi_{22}-1\right)-1$ close to zero. ${ }^{7}$ A refined analysis based on bifurcation theory is therefore required to determine whether E-stable SSEs exist near a steady state with $F^{\prime}(\hat{x})<-1$.

\section{Existence of E-stable SSEs}

We now prove that for the case $F^{\prime}(\hat{x})<-1$ E-stable Markov SSEs exist near the steady state.

Theorem 2 Assume $F^{\prime}(\hat{x})<-1$. For any neighborhood $\mathcal{U}$ of $(\hat{x}, \hat{x})$ there exists an E-stable $S S E\left(\bar{x}_{1}, \bar{x}_{2}\right) \in \mathcal{U}$.

The proof relies on a local bifurcation analysis of the differential equation (3) using the linearization (5), see e.g. Chapters 2 and 3 of (Wiggins 1990).

\footnotetext{
${ }^{7}$ (Chiappori, Geoffard, and Guesnerie 1992) show that a continuum of Markov SSEs exist near an indeterminate steady state using an argument based on limit transition probabilities for which $F^{\prime}(\hat{x})\left(\pi_{11}+\pi_{22}-1\right)-1$ is zero.
} 
The bifurcation arises when the linear part of the system has a zero eigenvalue, i.e. $\pi_{11}+\pi_{22}-1=\left(F^{\prime}(\hat{x})\right)^{-1}$. Treating $\pi_{22}$ as a fixed number we vary $\pi_{11}$ to achieve bifurcation. Letting

$$
\bar{\pi}_{11}=1+\left(F^{\prime}(\hat{x})\right)^{-1}-\pi_{22} \text { and } \nu=\pi_{11}-\bar{\pi}_{11},
$$

the bifurcation occurs at $\nu=0$. The system (3) is now written

$$
\begin{aligned}
& \dot{w}_{1}=\left(\bar{\pi}_{11}+\nu\right) F\left(\hat{x}+w_{1}\right)+\left(1-\bar{\pi}_{11}-\nu\right) F\left(\hat{x}+w_{2}\right)-w_{1}-\hat{x}, \\
& \dot{w}_{2}=\left(1-\pi_{22}\right) F\left(\hat{x}+w_{1}\right)+\pi_{22} F\left(\hat{x}+w_{2}\right)-w_{2}-\hat{x} .
\end{aligned}
$$

At $w_{1}=w_{2}=\nu=0$, the coefficient matrix of the linear part is $A=F^{\prime}(\hat{x}) \Pi-$ $I$, which has roots 0 and $F^{\prime}-1$, where $F^{\prime} \equiv F^{\prime}(\hat{x})$. The diagonalization of $A$ is given by $A=Q\left(\begin{array}{cc}0 & 0 \\ 0 & F^{\prime}-1\end{array}\right) Q^{-1}$ where $Q=\left(\begin{array}{ll}1 & 1 \\ a & 1\end{array}\right)$, with $a=\frac{\left(1-\pi_{22}\right) F^{\prime}}{1-\pi_{22} F^{\prime}}$.

Writing

$$
\left(\begin{array}{l}
y_{1} \\
y_{2}
\end{array}\right)=Q^{-1}\left(\begin{array}{l}
w_{1} \\
w_{2}
\end{array}\right)
$$

we obtain

$$
\dot{y}_{i}=G_{i}\left(y_{1}, y_{2}, \nu\right)
$$

for $i=1,2$, where

$$
\begin{aligned}
& G_{i}\left(y_{1}, y_{2}, \nu\right)= \\
& q^{i 1}\left[\left(\bar{\pi}_{11}+\nu\right) F\left(\hat{x}+y_{1}+y_{2}\right)+\left(1-\bar{\pi}_{11}-\nu\right) F\left(\hat{x}+a y_{1}+y_{2}\right)-y_{1}-y_{2}-\hat{x}\right] \\
& +q^{i 2}\left[\left(1-\pi_{22}\right) F\left(\hat{x}+y_{1}+y_{2}\right)+\pi_{22} F\left(\hat{x}+a y_{1}+y_{2}\right)-a y_{1}-y_{2}-\hat{x}\right] .
\end{aligned}
$$

Augmenting this system with $\dot{\nu}=0$ leads to a three-dimensional system for which the equations for $\dot{y}_{1}$ and $\dot{\nu}$ have zero linear parts and the equation for $\dot{y}_{2}$ has the linear part $\left(F^{\prime}-1\right) y_{2}$ which is obviously stable. We now use the center manifold theory, see e.g. pp. 194-203 of (Wiggins 1990). In particular, the system has an invariant center manifold which can be represented by a three times continuously differentiable function $y_{2}=h\left(y_{1}, \nu\right)$ with $h(0,0)=0$ and $D h(0,0)=0$. Local stability of the system is governed by local stability of the "projected system"

$$
\begin{aligned}
\dot{y}_{1} & =G_{1}\left(y_{1}, h\left(y_{1}, \nu\right), \nu\right), \\
\dot{\nu} & =0 .
\end{aligned}
$$


The second order expansions are

$$
\begin{aligned}
F\left(\hat{x}+y_{1}+y_{2}\right) & \doteq F(\hat{x})+F^{\prime} y_{1}+F^{\prime} y_{2}+\frac{1}{2} F^{\prime \prime}\left(y_{1}^{2}+y_{2}^{2}+2 y_{1} y_{2}\right), \\
F\left(\hat{x}+a y_{1}+y_{2}\right) & \doteq F(\hat{x})+F^{\prime} a y_{1}+F^{\prime} y_{2}+\frac{1}{2} F^{\prime \prime}\left(a^{2} y_{1}^{2}+y_{2}^{2}+2 a y_{1} y_{2}\right) \\
h\left(y_{1}, \nu\right) & =c y_{1}^{2}+d y_{1} \nu+f \nu^{2}+\mathcal{O}\left(\left\|\left(y_{1}, \nu\right)\right\|^{3}\right)
\end{aligned}
$$

where $F^{\prime \prime} \equiv F^{\prime \prime}(\hat{x})$ and $\doteq$ denotes equality up to $\mathcal{O}\left(\left\|\left(y_{1}, y_{2}\right)\right\|^{3}\right)$. It follows that on the center manifold the differential equation for $y_{1}$ can be written as

$$
\dot{y}_{1}=F^{\prime} \nu y_{1}+\frac{1}{2} \frac{F^{\prime \prime}}{F^{\prime}}(1+a) y_{1}^{2}+\mathcal{O}\left(\left\|\left(y_{1}, \nu\right)\right\|^{3}\right) .
$$

For the purposes of the theorem we are at liberty to choose $\pi_{22}$ so that $a \neq-1$ which we now assume. ${ }^{8}$

From (6) it is evident that the system exhibits a transcritical bifurcation at $\nu=0$. The SSEs are defined by the equations

$$
\begin{aligned}
& \bar{y}_{1}=\frac{2\left(F^{\prime}\right)^{2}}{F^{\prime \prime}(1+a)} \nu, \\
& \bar{y}_{2}=0 .
\end{aligned}
$$

In terms of the original variables we have

$$
w_{1}=\bar{y}_{1} \text { and } w_{2}=a \bar{y}_{1} .
$$

It follows that E-stable SSEs exist for $\nu<0$ in this case. If $\nu>0$ the SSEs are not E-stable and learning instead converges to the steady state.

We conclude with two supplementary remarks:

(1) The bifurcation analysis shows that there also exist E-unstable Markov SSEs near $\hat{x}$.

(2) A similar analysis can be conducted for $K$-state Markov SSEs, for $K>2$.

For the $K$-state case the linearization at the steady state is a higher dimensional analogue of (5) with the coefficient matrix $F^{\prime}(\hat{x}) \Pi-I$, where $\Pi$ and $I$ are $K \times K$ matrices. One of the roots of $F^{\prime}(\hat{x}) \Pi-I$ must be $F^{\prime}(\hat{x})-1$ and the transition matrix $\Pi$ can be chosen to yield one zero root and remaining roots $F^{\prime}(\hat{x}) \lambda_{i}-1$ with negative real parts. Thus an analogous result can be obtained for $K$-state SSEs.

\footnotetext{
${ }^{8} a=-1$ corresponds to the special case $\pi_{11}=\pi_{22}$, and this could also be analysed by looking at the third order terms. Since our object is to prove existence of E-stable SSEs for some choice of $\pi_{11}$ and $\pi_{22}$, it is not necessary to address this special case.
} 


\section{An Example}

We illustrate our result using a standard version of the Samuelson overlapping generations model of money. There is a constant population, and agents live for two periods. Each agent of generation $t$ supplies labor $n_{t}$ when young and consumes $c_{t+1}$ when old. Labor is the only input to production and output is perishable. There is a simple production function with one unit of labor yielding one unit of output. Agents save all of their income in the form of money, which they use to buy the consumption good when old. There is a constant stock $M=1$ of money.

The utility function of the representative agent of generation $t$ takes the form

$$
W=\frac{c_{t+1}^{1-\sigma}}{1-\sigma}-\frac{n_{t}^{1+\varepsilon}}{1+\varepsilon},
$$

where the parameters $\sigma, \varepsilon>0$. The budget constraints are

$$
p_{t} n_{t}=m_{t}, p_{t+1} c_{t+1}=m_{t},
$$

where $m_{t}$ denotes the money balance carried forward. The first order condition for maximization of expected utility is

$$
n_{t}^{\varepsilon}=E_{t}^{*}\left[\frac{p_{t}}{p_{t+1}}\left(\frac{p_{t} n_{t}}{p_{t+1}}\right)^{-\sigma}\right]
$$

or

$$
n_{t}^{\sigma+\varepsilon}=E_{t}^{*}\left(\frac{p_{t}}{p_{t+1}}\right)^{1-\sigma} .
$$

Here $E_{t}^{*}$ denotes the expectation held by agents at time $t$. In a rational expectations equilibrium this will be equal to the true mathematical conditional expectation. We assume that current price is observable and hence expectations are required for $p_{t+1}^{\sigma-1}$.

The market clearing condition is $m_{t}=1$, so that $n_{t}=1 / p_{t}$ and hence the temporary equilibrium is given by

$$
p_{t}^{-(1+\varepsilon)}=E_{t}^{*} p_{t+1}^{\sigma-1}
$$

To put this in the form (1) we transform variables to $x_{t}=p_{t}^{-(1+\varepsilon)}$ which yields the reduced form

$$
x_{t}=E_{t}^{*} x_{t+1}^{\phi}, \text { where } \phi=\frac{1-\sigma}{1+\varepsilon} .
$$




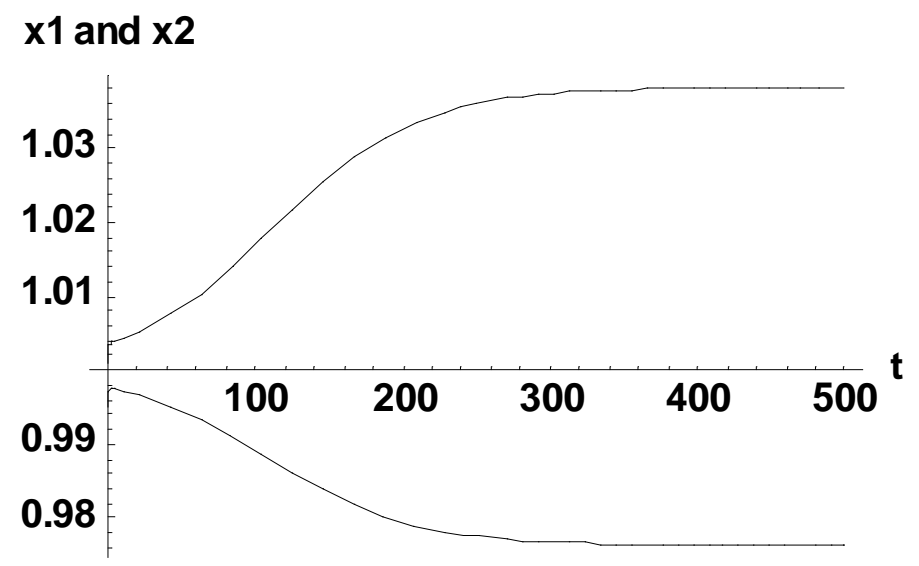

Figure 1: Convergence of adaptive learning to an SSE

Under adaptive learning the agents have a perceived law of motion in which the value of prices and hence of $x_{t}$ depend on the value of a two-state Markov sunspot variable $s_{t}$.

We are now in the standard setting with $F(x)=x^{\phi}$. The steady state is given by $\hat{x}=1$ and is indeterminate if $\phi<-1$ i.e. $\sigma>2+\varepsilon$. From our results we know that there then exist E-stable SSEs near the steady state. Note that such SSEs must satisfy $\pi_{22} \in\left[0,1+\phi^{-1}\right]$. E-stable SSEs occur for negative values of $\nu$. We illustrate the cases of E-stable SSEs and an E-stable steady state by simulating the differential equation (3). The parameter values are $\phi=-2, \bar{\pi}_{11}=0.1$ and $\pi_{22}=0.4$.

Figure 1 shows the case of convergence to an SSE when $\nu<0$. (For this simulation the value $\nu=-0.01$ was used.) In contrast, when $\nu>0$ we get convergence to the steady state. This is illustrated in Figure 2 for the choice $\nu=+0.01$.

\section{Conclusions}

This paper has provided sharp results on the issue of existence of learnable finite state Markov SSEs in a neighborhood of an indeterminate steady state for the general univariate model $x_{t}=E_{t}^{*} F\left(x_{t+1}\right)$. If the derivative $F^{\prime}(\hat{x})>1$ all such SSEs fail to be E-stable and hence are not learnable under adaptive learning. However, if $F^{\prime}(\hat{x})<-1$, learnable SSEs always exist sufficiently 


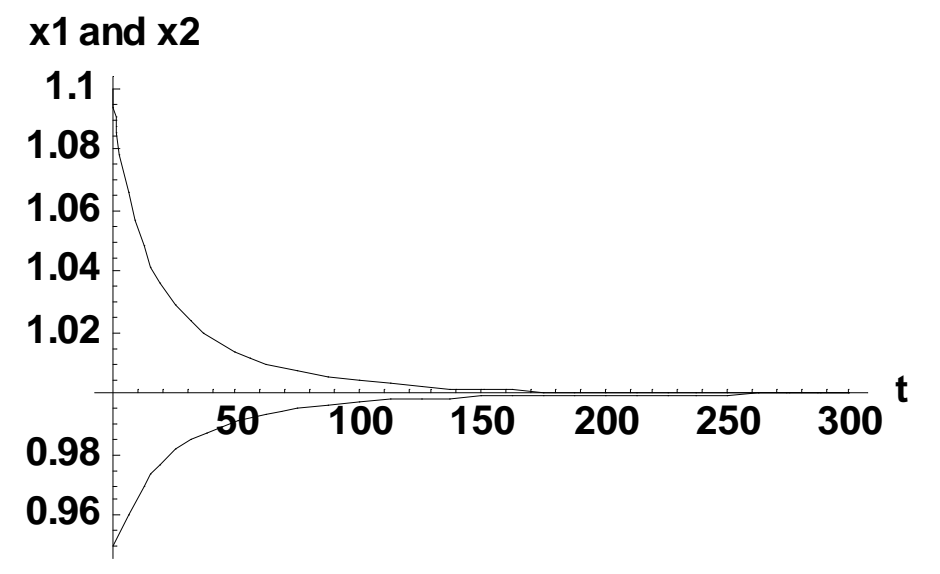

Figure 2: Convergence of adaptive learning to steady state

near the steady state.

These theoretical results suggest that it is important for applied researchers to determine the adaptive stability of sunspot equilibria near indeterminate steady states. Even though theoretical analysis may not be feasible in more complex models, it is usually possible to determine E-stability numerically using simulations. Both stable and unstable cases of Markov SSEs have been found to arise in applied models as witnessed by current work, see (Evans, Honkapohja, and Marimon 2001) and (Honkapohja and Mitra 2001). 


\section{References}

Azariadis, C. (1981): "Self-Fulfilling Prophecies," Journal of Economic Theory, 25, 380-396.

Benhabib, J., and R. E. Farmer (1999): "Indeterminacy and Sunspots in Macroeconomics," in (Taylor and Woodford 1999), chap. 6, pp. 387-448.

Cass, D., and K. Shell (1983): "Do Sunspots Matter?," Journal of Political Economy, 91, 193-227.

Chiappori, P., and R. Guesnerie (1991): "Sunspot Equilibria in Sequential Market Models," in (Hildenbrand and Sonnenschein 1991), pp. $1683-1762$.

Chiappori, P. A., P.-Y. Geoffard, and R. Guesnerie (1992): "Sunspot Fluctuations around a Steady State: The Case of Multidimensional, One-Step Forward Looking Economic Models," Econometrica, 60, 1097-1126.

Desgranges, G., and G. Negroni (2001): "Expectations Coordination on a Sunspot Equilibrium: an Eductive Approach," Macroeconomic Dynamics, forthcoming.

Evans, G. W., and S. Honkapohja (1994): "On the Local Stability of Sunspot Equilibria under Adaptive Learning Rules," Journal of Economic Theory, 64, 142-161.

- (2001): Learning and Expectations in Macroeconomics. Princeton University Press, Princeton, New Jersey.

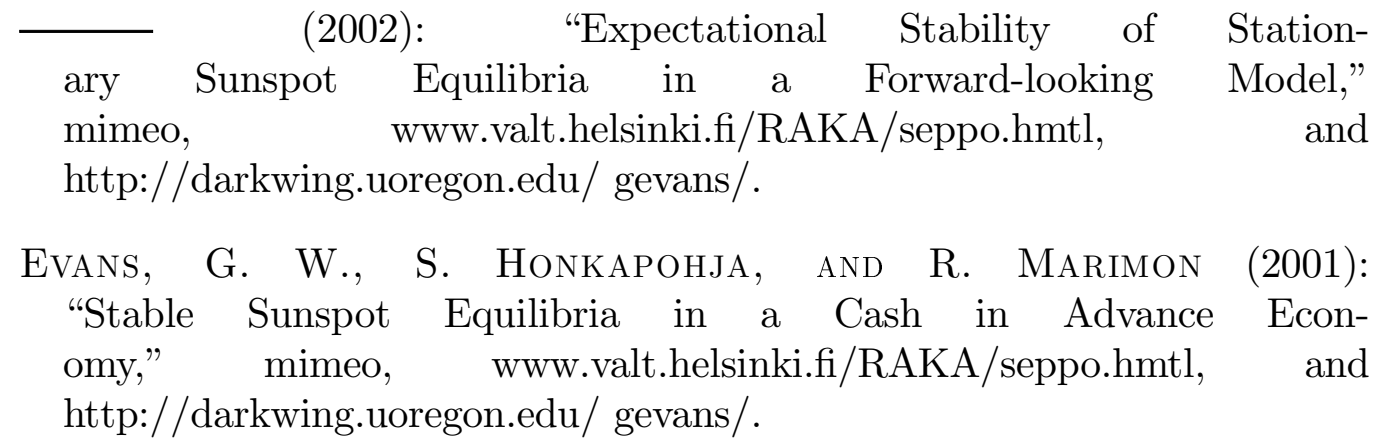


Evans, G. W., S. Honkapohja, and P. Romer (1998): "Growth Cycles," American Economic Review, 88, 495-515.

Farmer, R. E. (1999): The Economics of Self-Fulfilling Prophecies, Second edition. MIT Press, Cambridge, Mass.

Guesnerie, R., and M. Woodford (1992): "Endogenous Fluctuations," in (Laffont 1992), chap. 6, pp. 289-412.

Hildenbrand, W., And H. Sonnenschein (eds.) (1991): Handbook of Mathematical Economics, Vol. IV. North-Holland, Amsterdam.

Honkapohja, S., And K. Mitra (2001): "Are Non-Fundamental Equilibria Learnable in Models of Monetary Policy?," Working paper, www.valt.helsinki.fi/RAKA/seppo.hmtl.

Howitt, P., and R. P. McAfee (1992): "Animal Spirits," American Economic Review, 82, 493-507.

Laffont, J.-J. (ed.) (1992): Advances in Economic Theory: Sixth World Congress. Volume 2. Cambridge University Press, Cambridge, UK.

SheLL, K. (1977): "Monnaie et Allocation Intertemporelle," Working paper, CNRS Seminaire de E.Malinvaud, Paris.

TAylor, J., And M. Woodford (eds.) (1999): Handbook of Macroeconomics, Volume 1. Elsevier, Amsterdam.

Wiggins, S. (1990): Introduction to Applied Nonlinear Dynamical Systems and Chaos. Springer-Verlag, Berlin.

Woodford, M. (1990): "Learning to Believe in Sunspots," Econometrica, $58,277-307$. 